\section{Summary}

Complete development of Cryptosporidium in vitro : applications Protozoan parasites of the genus Cryptosporidium cause a short term, flu-like, gastrointestinal illness in immunocompetent persons and a severe, persistent life threatening diarrhea in immunodeficient patients. No effective therapy is available for the treatment of crytosporidiosis in the immunodeficient host. Complete development (from sporozoite to sporulated oocyst) of Cryptosporidium was achieved in cultured human colon cancer cells (CACO2 cells) which in vitro acquired the enterocyte's characteristics. The growth of Cryptosporidium in CACO2 cells provides a novel means for research in the field of drug screening. Spiramycin seemed to have some effects in vivo and at first, the authors tested this drug. Initially, spiramycin's toxicity and its penetration in CACO2 cells were evaluated; then its activity on cultured Cryptosporidium was tested. Spiramycin revealed itself to be completely ineffective. The same negative results were observed with eflornithine, mefloquine, pentamidine, mefloxacine, norfloxacine, pefloxacine and imipenem.

\section{ADRESSE}

A. Datry: maître de conférences des universités, praticien hospitalier. M. Danis : professeur des universités, praticien hospitalier. M. Gentilini : professeur des universités, chef de service. Département de parasitologie, médecine tropicale et santé publique et INSERM U. 313, hôpital de la Salpêtrière, boulevard de l'Hôpital, 75013 Paris, France.

\section{TRÉS A PART}

\title{
Développement complet de Cryptosporidium en culture cellulaire : applications
}

\author{
Annick Datry, Martin Danis, Marc Gentilini
}

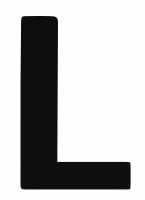

es protozoaires du genre Cryptosporidium (Phylum Apicomplexa, sous-ordre Eimeriina) sont des coccidies de petite taille (2 à $6 \mu \mathrm{m})$, se développant dans les cellules épithéliales de l'intestin de nombreux animaux et de l'homme [1].

Cryptosporidium est décrit pour la première fois en 1907 par Tyzzer dans les cryptes gastriques d'une souris ; ce n'est qu'en 1955 que Slavin lui reconnaît un rôle pathogène chez le dindon [2, 3]. Depuis cette date, la cryptosporidiose est devenue une étiologie importante de gastro-entérite et de diarrhée dans de nombreuses espèces animales, spécialement les veaux, les agneaux, les chèvres et l'homme [4].

De 1976 à 1981, les sept premiers cas humains de cryptosporidiose sont rapportés [5, 6]. Cinq de ces patients sont immunodéprimés et Cryptosporidium est considéré comme un protozoaire opportuniste rare chez l'homme. De 1981 à 1982, 47 autres cas sont recensés par le $\mathrm{CDC}$, la plupart survenant chez des patients atteints de $\operatorname{SIDA}[7,8]$. Dès lors, le nombre de cas de cryptosporidiose humaine ne cesse de croître avec l'ascension du SIDA.

La cryptosporidiose des immunodéprimés, et surtout ceux atteints de SIDA, diarrhée sévère, prolongée, parfois cholériforme, contraste avec la courte gastro-entérite, s'éteignant d'elle-même, du sujet immunocom- pétent $[9,10]$. Les techniques simples mises en œuvre pour la détection des oocystes de Cryptosporidium permettent de donner une étiologie aux diarrhées jusqu'alors inexpliquées de l'enfant, surtout en zone tropicale, avec une fréquence parfois supérieure à $30 \%$ [11-14].

Des infections respiratoires et biliaires à Cryptosporidium montrent que ce protozoaire n'est pas toujours confiné au tractus digestif $[15,16]$.

Les infections expérimentales prouvent qu'il n'existe pas de spécificité d'hôte et le mode de contamination s'effectue par ingestion d'oocystes sporulés et infectieux dès leur émission dans les selles [17].

L'homme s'infeste par l'intermédiaire des animaux infectés, des hommes malades ou porteurs sains, ou par des eaux polluées [18-20].

L'absence de molécules actives sur Cryptosporidium crée actuellement une difficulté majeure. Les multiples essais thérapeutiques restent vains chez l'homme [21, 22]. Les oocystes de Cryptosporidium résistent aux désinfectants usuels, posant le problème de désinfection des endoscopes et des eaux polluées [23].

Le développement complet de Cryptosporidium est réalisé dans des embryons de poulet, puis sur cellules fotales humaines de poumon[24, 25].

A la lumière de ces travaux, nous avons obtenu le cycle complet de Cryptosporidium sur hépatocytes et 

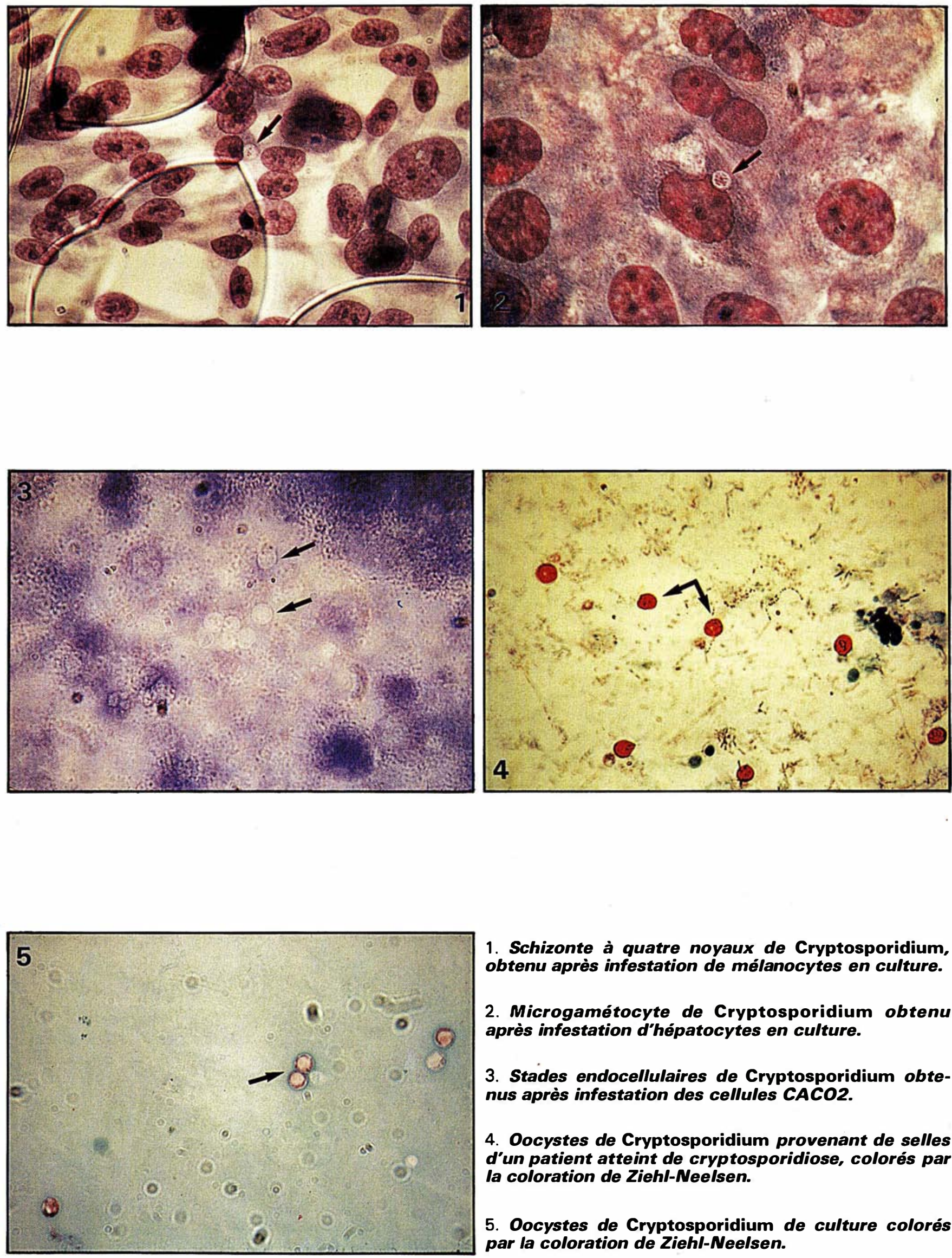

1. Schizonte à quatre noyaux de Cryptosporidium, obtenu après infestation de mélanocytes en culture.

2. Microgamétocyte de Cryptosporidium obtenu après infestation d'hépatocytes en culture.

3. Stades endocellulaires de Cryptosporidium obtenus après infestation des cellules CACO2.

4. Oocystes de Cryptosporidium provenant de selles d'un patient atteint de cryptosporidiose, colorés par la coloration de Ziehl-Neelsen.

5. Oocystes de Cryptosporidium de culture colorés par la coloration de Ziehl-Neelsen. 
sur une lignée cellulaire issue de cancer colique humain (lignée CACO2 obtenue de J. Fogh, Sloan Kettering Institute for Cancer Research, Rye, NY, États-Unis) [26]. Les cellules CACO2 acquièrent spontanément en culture une différenciation de type entérocytaire. Ce modèle in vitro donne une bonne corrélation avec le cycle humain et permet d'apprécier l'activité de molécules potentiellement actives chez l'homme [27, 28]. La culture de Cryptosporidium in vitro permettrait de déterminer avec précision la biologie du parasite intracellulaire en l'absence d'autres éléments pathogènes.

\section{Développement complet de Cryptosporidium in vitro}

\section{Méthodes d'étude.}

(1) Obtention d'oocystes purifiés de Cryptosporidium: les oocystes de Cryptosporidium proviennent des selles de patients atteints de SIDA et qui présentent une cryptosporidiose chronique. Les selles sont stockées à $4^{\circ} \mathrm{C}$ dans une solution à $2,5 \%$ de bichromate de potassium $\left(\mathrm{K}_{2} \mathrm{CR}_{2} \mathrm{O}_{7}\right)$ pendant au moins trois mois. Les oocystes sont ensuite purifiés et concentrés par flottation dans une solution sucrée de Sheather et lavés trois fois par centrifugation dans de l'eau distillée stérile. La totalité des levures et bactéries est éliminée par mise en suspension des oocystes dans une solution d'hypochlorite de sodium à $1,75 \%$ pendant une heure.

(2) Obtention des sporozoïtes. Après plusieurs lavages, les oocystes sont incubés une heure en agitation à $37^{\circ} \mathrm{C}$ dans de la trypsine EDTA $1 \mathrm{X}$ taurocholate. Les sporozoïtes libérés sont lavés deux fois dans un tampon phosphate (PBS, $\mathrm{pH}=7,2$ ) et resuspendus dans le milieu de culture (MEM Dulbeco ou RPMI).

(3) Infestation des cellules. 150000 sporozoïtes sont ajoutés sur quatre milieux cellulaires cultivés en monocouche dans des boîtes de Petri Falcon $35 \mathrm{~min}$ contenant $1,5 \mathrm{ml}$ de milieu: (a) des cellules de mélanome (ATCC n' ${ }^{\circ}$ CRL 1585) cultivées en milieu RPMI avec $10 \%$ de SVF (sérum de veau fotal); (b) des primocultures d'hépatocytes humains un milieu MEM avec $10 \%$ de SVF; (c) la lignée cellulaire CACO2 qui acquière in vitro les caractéristiques de l'entérocyte et est entretenue dans un milieu MEM Dulbeco avec $20 \%$ de SVF.

- Résultats et discussion (Tableau I) Sur les cellules de mélanome, le cycle complet n'a pu être obtenu. Seuls des trophozoïtes et des schizontes sont observés.

Dans les hépatocytes de rat ou humains, des schizontes à huit et à quatre noyaux apparaissent dès les premières 24 heures et les oocystes, dès le $3^{\mathrm{e}}$ jour avec un pic d'émission aux $5^{e}$ et $6^{e}$ jours.

Sur les cellules de la lignée CACO2, il est difficile d'apprécier les stades endocellulaires; toutefois, le cycle complet de Cryptosporidium est aussi obtenu puisque des oocystes apparaissent dans les milieux de culture le $3^{\mathrm{e}}$ jour, avec un pic d'émission aux $5^{\mathrm{e}}$ et $6^{\mathrm{e}}$ jours, beaucoup plus important que sur les autres supports cellulaires.

Les oocystes de culture obtenus sur les hépatocytes et sur les cellules de la lignée CACO2 sont traités par le mélange trypsine-taurocholate et sont inoculés à des cellules CACO2. L'obtention d'un nouveau cycle prouve la viabilité de ces oocystes de culture.

La spécificité du sporozoïte semble faible, puisque Cryptosporidium peut se développer sur des cellules très variées [24-26].

Cependant, le développement intracellulaire du protozoaire présente de grandes variations. La lignée cellulaire CACO2 permet un rendement

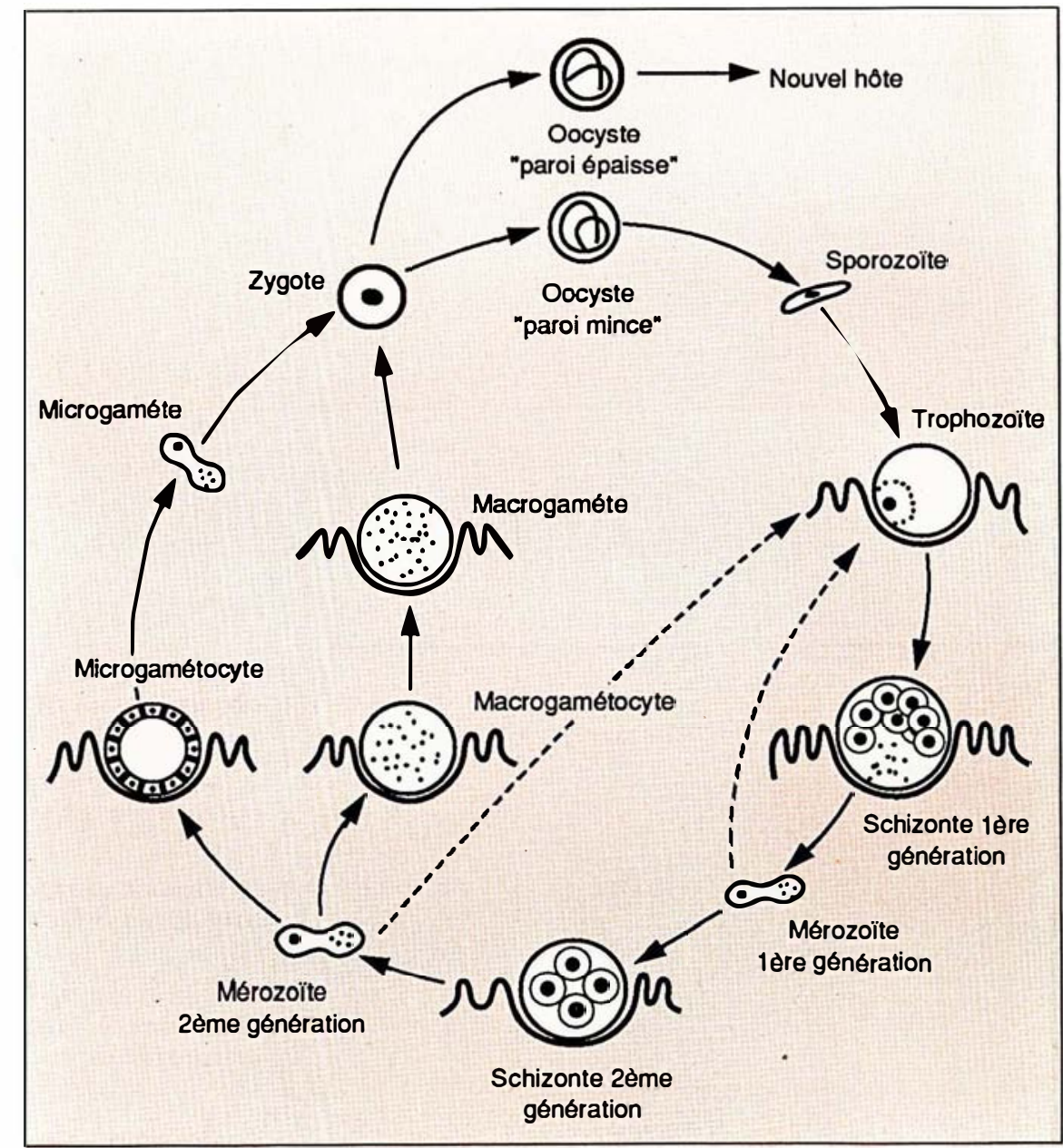

Figure 1. Schéma du cycle parasitaire de Cryptosporidium. 


\begin{tabular}{|c|c|c|c|c|c|c|c|c|c|c|c|c|c|c|c|c|}
\hline \multicolumn{17}{|c|}{$\begin{array}{l}\text { APPRÉCIATION SEMI-QUANTITATIVE DES FORMES PARASITAIRES OBTENUES } \\
\text { AVEC LES DIFFÉRENTS SUPPORTS CELLULAIRES EN FONCTION DU TEMPS }\end{array}$} \\
\hline \multirow{2}{*}{$\begin{array}{c}\text { Cellule } \\
\text { Stades } \\
\text { parasitaires }\end{array}$} & \multicolumn{4}{|c|}{ Mélanome } & \multicolumn{4}{|c|}{ Hépatocyte de rat } & \multicolumn{4}{|c|}{ Hépatocyte humain } & \multicolumn{4}{|c|}{ Lignée CACO2 } \\
\hline & SI & SII & M.m & O० & SI & SII & M.m & 00 & SI & SII & M.m & OO & SI & SII & M.m & O० \\
\hline J1 & 0 & 0 & 0 & 0 & $+/-$ & $+/-$ & 0 & 0 & $+/-$ & $+/-$ & 0 & 0 & - & - & - & 0 \\
\hline $\mathrm{J} 2$ & $+/-$ & $+1-$ & 0 & 0 & ++ & ++ & ++ & 0 & + & + & 0 & 0 & - & - & - & 0 \\
\hline J3 & $+/-$ & $+1-$ & 0 & 0 & ++ & ++ & ++ & + & + & + & + & + & - & - & - & + \\
\hline$J 4$ & 0 & $+/-$ & 0 & 0 & + & + & + & + & 0 & + & + & + & - & - & - & ++ \\
\hline J5 & 0 & $+1-$ & 0 & 0 & + & + & + & ++ & 0 & + & + & + & - & - & - & +++ \\
\hline J6 & 0 & 0 & 0 & 0 & 0 & 0 & + & ++ & 0 & 0 & 0 & + & - & - & - & +++ \\
\hline $\mathrm{J7}$ & 0 & 0 & 0 & 0 & 0 & 0 & 0 & + & 0 & 0 & 0 & 0 & - & - & - & ++ \\
\hline
\end{tabular}

$0=$ absent $;+/-=$ rare $;+=$ faible $;++=$ nombreux $;+++=$ très nombreux $; S I=$ schizonte de 1 re génération; SII $=$ schizonte de $2^{e}$ génération $; M \cdot m=$ macro- et microgamétocytes; $00=$ oocystes.

\section{RÉFÉRENCES}

1. Tzipori S. Cryptosporidiosis in animals and humans. Microbiol Rev 1983 ; $47: 84-96$.

2. Slavin D. Cryptosporidium meleagridis (sp. nov.). J Comp Pathol Ther 1955 ; 70 : 5928.

3. Tyzzer EE. A sporozoan found in the peptic glands of the common mouse. Proc Soc Exp Biol Med 1907 ; 5 : 12-3.

4. Schultz MG. Emerging zoonoses. N Engl J Med 1983 ; 308 : 1285-6.

5. Meisel JL, Perera DR, MeligroC, Rubin CE. Overwhelming watery diarrhea associated with a Cryptosporidium in an immunosuppressed patient. Gastroenterology $1976 ; 70$ : 1156-60.

6. Weisburger WR, Hutcheon DF, Yardley JH, Roche JC, Hillis WD, Charache P. Cryptosporidiosis in an immunosuppressed renal transplant recipient with IgA deficiency. Am J Clin Pathol 1979 ; 72 : 473-8.

7. Centers for Disease Control. Assessment of chemotherapy of males with acquired immune deficiency syndrome (AIDS). MMWR 1982 ; 31 : 589-92

8. Navin TR, Juranek DD. Cryptosporidiosis : clinical epidemiologic and parasitologic review. Rev Infect Dis 1984; 6 : 313-27.

9. Andreani T, Le Charpentier Y, Brouet JC, et al. Acquired immunodeficiency with intestinal cryptosporidiosis. Lancet 1983 ; i : 1187. 91.

$\mathrm{m} / \mathrm{s} n^{\circ} 10$ vol. 5 , décembre 89
10. BabbRR, Differding JT, Trollope ML Cryptosporidia enteritis in a healthy professional athlete. Am J Gastroenterol 1982; 77 : 833-4.

11. Centers for Disease Control. Cryptosporidiosis among children attending day care centers: Georgie, Pennsylvania, Michigan, California, New Mexico. MMWR 1984; 33 : 599-601.

12. Cross JH, AlcantaraA, Alquiza L Zaraspe G, Ranoa C. Cryptosporidiosis in Phillipine children. South Asian J Trop Med Pub Health 1985 ; 16 : 257.

13. Guderian RH, Sandoval CA, MacKenzie CD. Cryptosporidiosis in Equatorian children with acute diarrhea. J Trop Pediat 1986 ; $32: 290$.

14. Shahid NS, Rahman ASMH, Ander son BC. Cryptosporidiosis in Bangladesh. B Med J 1985; 290 : 114-115.

15. BlagburnRS, Kelsey P, Perrone T Dickersin R, Laquarglia M, Ferruci J. Cytomegalovirus and Cryptosporidium associated a calculous gangrenous cholecystis. $\mathrm{Am} \mathrm{J}$ Med 1984; 76 : 1118-23.

16. Brady EM, Margolis ML, Korzeniowski DH. Pulmonary cryptosporidiosis in AIDS. JAMA 1984 ; 252 : 89-90.

17. Current WL. Human cryptosporidiosis $N$ Engl J Med 1983 ; 309 : 1326-7. important d'oocystes. En raison de sa capacité à différencier une bordure en brosse, cette lignée, proche de la cellule naturellement infestée, semble être un modèle d'étude intéressant.

\section{Applications}

Évaluation in vitro de l'efficacité de molécules sur Cryptosporidium $s p$ [27]. Le choix des molécules testées est réalisé en fonction de leur activité partielle chez l'homme ou chez l'animal. La spiramycine est la première molécule à montrer quelques effets sur la cryptosporidiose humaine [28]. L'évaluation in vitro de l'activité de cette molécule sur Cryptosporidium permet la mise au point d'une méthode de criblage primaire de molécules potentiellement actives sur Cryptosporidium $s p$.

Nos résultats démontrent cependant que, in vitro, aucune efficacité de la spiramycine sur l'ensemble des stades de développement du parasite ne peut être mise en évidence, alors même que la pénétration intracellulaire de l'antibiotique apparaît satisfaisante (figure 2, p. 766). Nous n'avons pas été plus heureux avec d'autres molécules testées dans les mêmes conditions: eflornithine, méfloquine, pentamidine, méfloxacine, péfloxacine, imipénème. 


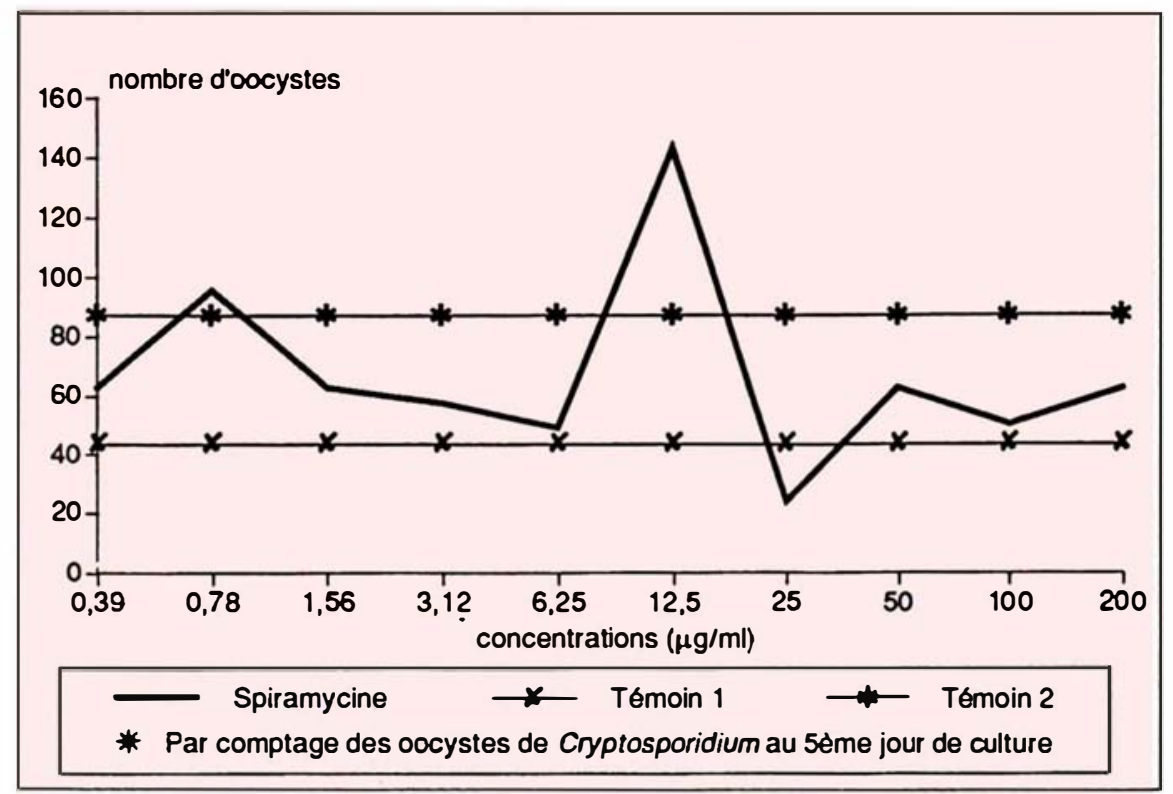

Figure 2. Évaluation de l'activité in vitro de la spiramycine sur Cryptosporidium*.

\section{Conclusion}

Le développement de Cryptosporidium sur entérocytes in vitro offre aux expérimentateurs un excellent modèle expérimental puisqu'il s'agit de la localisation habituelle du parasite chez l'homme. Il permet l'étude de molécules potentiellement actives sur Cryptosporidium, mais aussi l'obtention d'oocystes purifiés, source d'antigènes commodes pour affiner les techniques sérologiques actuelles. Enfin, la réussite de ce cycle in vitro pourra peut-être élucider le mécanisme intime entre le parasite et la cellule hôte, éclaircir la physiopathologie de la diarrhée cholériforme des patients atteints du SIDA et définir avec exactitude le cycle de ce protozoaire

\section{RÉFÉRENCES}

18. Anderson BC, Donnedelinger T, Wilkins MR, Smith J. Cryptosporidiosis in a veterinary student. JAMA $1980 ; 182$ : 408-9.

19. Blagburn B, Current WL. Accidental infection of a researcher with human Cryptosporidium. J Infect Dis 1983; $148: 772-773$.

20. Rush BA, Chapman PP, Ineson RW. Cryptosporidium and drinking water. Lancet
21. Hart A, Baxby D. Management of Cryptosporidiosis. J Antimicrob Chemother 1985 ; $15: 3-4$.

22. Soave $\mathbf{R}$. Therapy and prevention of coccidiosis. In : Leive L, Schlessenger D, eds. Microbiology. Washington: Am Soc Microbiol $1984 ; 232$.

23. Anderson BC. Most heat inactivation of Cryptosporidium sp. Am J Pub Health 1985 ; $75: 1433-4$.

24. Current WL, Long PL. Development of human and calf Cryptosporidium in chicken embryos. J Infect Dis 1983 ; 148 : 1108-13.

25. Current WL, Haynes TH. Complete development of Cryptosporidium in cell culture. Science 1984 ; 224 : 603-5.

26. Datry A, Bretagne S, Zweibaum A, Mellouck S, Mazier D, Monjour L. Complete development of Cryptosporidium. Second International Conference on Acquired Immunodeficiency Syndrome (AIDS). Paris, 1986.

27. Datry A, Brandicourt O, Bellane $C$, Pajot N, Morin C, Danis M, Gentilini M. Effects of drugs on Cryptosporidium in cell culture in vitro. IVth International Conference on AIDS. Stockholm, 1988.

28. Portnoy D, Whiteside ME, Buckley E, Mac Leod CL. Treatment of intestinal cryptosporidiosis with spiramycin. Ann Intern Med 1984; 101 : 202-204. 